\title{
Quark masses in two-flavor QCD
}

\author{
Michael Creutz*† \\ Brookhaven National Laboratory \\ E-mail: creutz@bnl.gov
}

Considered as a function of the quark mases, two-flavor QCD depends on three parameters, including one that is $\mathrm{CP}$ violating. As the masses vary to unphysical values, regions of both first- and second-order phase transitions are expected. For non-degenerate quarks, non-perturbative effects leave individual quark mass ratios with a renormalization scheme dependence. This complicates matching lattice results with perturbative schemes and clarifies the tautology with attacking the strong CP problem via a vanishing up quark mass.

International Workshop on QCD Green's Functions, Confinement and Phenomenology 5-9 September 2011

Trento, Italy

\footnotetext{
*Speaker.

$\dagger$ I am grateful to the award I received from Alexander von Humboldt Foundation to visit the University of Mainz and which partially supported my attendence to this meeting. This manuscript has been authored under contract number DE-AC02-98CH10886 with the U.S. Department of Energy. Accordingly, the U.S. Government retains a non-exclusive, royalty-free license to publish or reproduce the published form of this contribution, or allow others to do so, for U.S. Government purposes.
} 


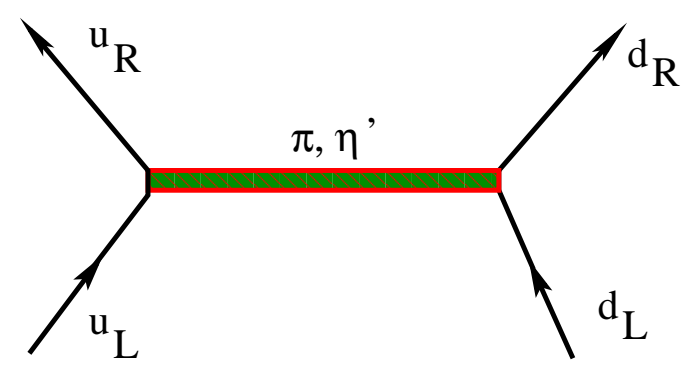

Figure 1: Because of the anomaly, spin-flip scattering of massless up and down quarks does not vanish.

\section{Introduction}

At the previous meeting in this series [1] I discussed the fascinating physics arising from the interplay of the three ways chiral symmetry is broken in QCD. These are (1) the spontaneous breaking responsible for the lightness of pions, (2) the breaking of the singlet axial $U(1)$ symmetry by the anomaly, and (3) the explicit breaking of chiral symmetry by the quark masses. For simplicity, that discussion was restricted to degenerate quarks. Here I move on to some interesting generalizations that occur when the quarks are no longer degenerate. Since the number of parameters grows with the number of flavors, I concentrate here on the two flavor theory and consider what happens when the quark masses are varied from their physical values. As a function of the these parameters a rather intricate phase diagram emerges, displaying both first and second order phase transitions. Much of this talk is adapted from the more detailed treatments in Refs. [2] and [3].

To begin, let me remind you of the expected behavior of two flavor QCD in the limit of massless quarks. Because of confinement and dimensional transmutation, this theory should possess several massive states, including the proton, neutron, eta prime, and glueballs. In addition, spontaneous chiral symmetry breaking should give rise to three massless pions as Goldstone bosons. In this picture both the eta prime and the neutral pion are composites of distinct mixtures of $\bar{u} u$ and $\bar{d} d$ quarks. The eta prime, defined as the lightest isosinglet pseudoscalar, also has a contribution from purely gluonic constituents. The latter are related to the anomaly and the fact that the $\pi_{0}$ and the $\eta^{\prime}$ are not degenerate.

In this theory, consider a hypothetical quark-quark scattering experiment, as sketched in Fig. 1. This represents spin flip scattering of an up quark against a down quark. Exchanges of both the neutral pion and the eta prime can contribute to this process. Because these particles are nondegenerate, their contributions cannot cancel. Therefore, the spin-flip four point function does not vanish. Were it not for the anomaly, the two exchanges could cancel.

Now turn on a small down quark mass. Take the diagram in Fig. 1 and close the down quark lines into a loop with a mass insertion as shown in Fig. 2. This provides a mechanism for mixing the left and right handed up quark, i.e. the up quark develops an effective mass. Starting with a vanishing up quark mass, the mass ration $\frac{m_{u}}{m_{d}}$ becomes renormalized by non-perturbative effects. Except in the isospin limit, quark mass ratios will not be renormalization group invariant. Since lattice gauge simulations include all non-perturbative physics, this effect is automatically present in such calculations.

This trivial observation is rather old and is often discussed in terms of instanton physics $[4,5,6$, 


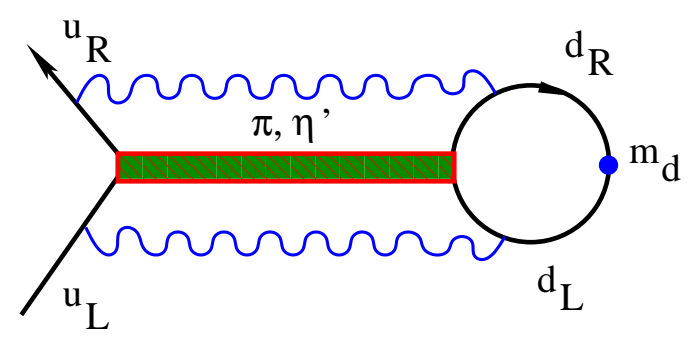

Figure 2: A small down quark mass induces an additive shift in the up quark mass through pseudoscalar meson exchange.

7]. Note that only the last of these references appears in a refereed journal, more than twenty years after the first. This is a consequence of an intense consternation from the perturbative community based on the lore that spin flip processes are suppressed in the massless limit.

This renormalization of quark mass ratios is an effect not seen in conventional perturbative schemes, such as $\overline{M S}$. The consequences have therefore been quite controversial. Mass renormalization is not flavor blind, and a mass independent renormalization scheme is problematic. The $\overline{M S}$ scheme is not a complete regulator since it ignores such non-perturbative effects. The crucial conclusion here is that when the up and down quarks are not degenerate, then attempts to match masses obtained from lattice calculations with perturbative results are inherently meaningless. I am not criticizing $\overline{M S}$ as a perturbative regulator; rather, the lattice and perturbative calculations involve different physics and should not be compared.

\section{Specific critiques}

The above observations raise frequent objections. At the simplest level, one might try to claim that the concept of $m=0$ corresponds to the bare mass rather than some running quantity. The problem with this is that the bare quark masses always vanish. The renormalization group tells us that as one approaches the continuum limit

$$
m_{0} \propto g_{0}^{\gamma_{0} / \beta_{0}}\left(1+O\left(g_{o}^{2}\right)\right)
$$

with the known coefficients

$$
\begin{aligned}
& \beta_{0}=\frac{11-2 n_{f} / 3}{(4 \pi)^{2}} \\
& \gamma_{0}=\frac{8}{(4 \pi)^{2}} .
\end{aligned}
$$

The asymptotic freedom result that the bare coupling $g_{0}$ goes to zero in the continuum limit then immediately implies $m_{0} \rightarrow 0$. To talk about quark masses as non-vanishing quantities, it is necessary to define them using some finite scale.

A more sophisticated complaint is that one has the option to use a mass independent regularization scheme. In the renormalization group equation for the mass

$$
a \frac{d m_{i}}{d a}=\gamma(g) m_{i}
$$

only the leading perturbative term in $\gamma(g)$ is scheme independent. If one requires that $\gamma(g)$ is independent of any of the quark masses, then one automatically obtains

$$
\frac{m_{i}}{m_{j}}=\text { constant. }
$$


Indeed, such a regularization is technically allowed, but it hides the above off-diagonal $m_{d}$ effect on $m_{u}$. There is no guarantee that quark mass ratios are independent of scheme, and the lattice, as usually implemented, is itself not a mass independent scheme. This makes it quite obscure how to do a matching with lattice results. To be more specific, when $m_{u}$ is different from $m_{d}$, isospin is broken explicitly and the charged pion mass differs from that of its neutral partner. A straightforward effective Lagrangian analysis relates the ratio of pion masses to the quark masses with the result

$$
\frac{m_{\pi^{0}}^{2}}{m_{\pi^{ \pm}}^{2}}=1-O\left(\frac{\left(m_{u}-m_{d}\right)^{2}}{\left(m_{u}+m_{d}\right) \Lambda_{q c d}}\right)
$$

As an immediate consequence, if one holds the quark masses fixed, then the physical hadronic mass ratios will be scale dependent. Conversely, if one holds the hadron mass ratios fixed, as usually done in lattice simulations, then the quark mass ratios must be scale dependent.

At this point advocates of the matching process frequently suggest doing the comparison at some high energy, say $100 \mathrm{GeV}$, where instantons are exponentially suppressed and irrelevant. This does not resolve the issue for several reasons. First the lattice simulations are not done at such small scales and the instanton effects must be included. Furthermore, the asymptotic freedom result

$$
1 / g^{2} \sim \log (\mu) \sim \log (1 / a)
$$

shows that the exponential suppression in $1 / g^{2}$ is actually only a power law suppression in the scale. One can easily estimate the size of these effects from the renormalization group, which tells us that

$$
m_{\eta^{\prime}} \propto \frac{1}{a} e^{-1 /\left(2 \beta_{0} g^{2}\right)} g^{-\beta_{1} / \beta_{0}^{2}} \nrightarrow \rightarrow 0 .
$$

The uncertainty in the up quark mass is proportional to this mass as well as being proportional to $m_{d}-m_{u}$. Thus the expected order of the up quark mass shift at a scale of a few $\mathrm{GeV}$ is

$$
\Delta m_{u}(\mu) \sim \frac{\left(m_{\eta^{\prime}}-m_{\pi_{0}}\right)\left(m_{d}-m_{u}\right)}{\Lambda_{q c d}}=O(1 \mathrm{MeV}) .
$$

This is a number comparable in size to the quoted lattice masses $[8,9,10]$.

In this context it is important to note that the exponent in Eq. (2.7), $\frac{8 \pi^{2}}{\left(11-2 n_{f} / 3\right) g^{2}}$, is considerably smaller than the classical instanton action $\frac{8 \pi^{2}}{g^{2}}$. This emphasizes that the relevant topological excitations need to be considered above the quantum, not the classical vacuum. Calculations based on the classical instanton solution strongly underestimate these effects. The renormalization group gives the correct suppression.

\section{General masses in two flavor QCD}

I now arrive at the main topic of this talk, the most general mass parameters for two flavor QCD. A mass term should be a dimension-three Hermitean quadratic form in the quark fields. As well it should be Lorentz invariant and electrically neutral. Based on these criteria, the most general expression is

$$
m_{1} \bar{\psi} \psi+m_{2} \bar{\psi} \tau_{3} \psi+i m_{3} \bar{\psi} \gamma_{5} \psi+i m_{4} \bar{\psi} \tau_{3} \gamma_{5} \psi
$$


Conventionally one might refer to these four terms with $m_{1}$ representing the average quark mass, $m_{2}$ the up-down mass difference, and $m_{3}$ a possible $\mathrm{CP}$ violating term related to the Theta parameter. Finally $m_{4}$ represents what is sometimes called a "twisted mass."

These four mass parameters are not independent. Consider a flavored chiral rotation of form $\psi \rightarrow e^{i \theta \tau_{3} \gamma_{5}} \psi$. Under this the various quadratic forms transform as

$$
\begin{aligned}
& \bar{\psi} \psi \quad \rightarrow \cos (\theta) \quad \bar{\psi} \psi \quad+\sin (\theta) \quad i \bar{\psi} \gamma_{5} \tau_{3} \psi \\
& \bar{\psi} \tau_{3} \psi \rightarrow \cos (\theta) \quad \bar{\psi} \tau_{3} \psi \quad+\sin (\theta) \quad i \bar{\psi} \gamma_{5} \psi \\
& i \bar{\psi} \gamma_{5} \psi \quad \rightarrow \cos (\theta) \quad i \bar{\psi} \gamma_{5} \psi \quad-\sin (\theta) \quad \bar{\psi} \tau_{3} \psi \\
& i \bar{\psi} \tau_{3} \gamma_{5} \psi \rightarrow \cos (\theta) \quad i \bar{\psi} \tau_{3} \gamma_{5} \psi \quad-\quad \sin (\theta) \quad \bar{\psi} \psi
\end{aligned}
$$

This rotation mixes $m_{1} \leftrightarrow m_{4}$ and $m_{2} \leftrightarrow m_{3}$. What is essentially a change of variables allows one to select any one of the $m_{i}$ to vanish and a second to be positive.

The conventional choice is to take $m_{4}=0$ and then use $m_{1}>0$ for the average quark mass and $m_{2}$ for the quark mass difference. The CP odd term proportional to $m_{3}$ is related to the Theta parameter and will be discussed further momentarily.

An alternative choice is to select $m_{1}=0$ and use $m_{4}>0$ as the average quark mass. Then the quark mass difference moves to the $m_{3}$ term and $m_{2}$ encodes the CP violation. This is the choice used for "twisted mass" lattice simulations. The primary motivation lies with certain lattice artifacts which depend on the twist. These are minimized with this choice [11,12].

It is important to recognize that the choice between these options is purely a convention and the continuum physics is equivalent between them. For the following discussion I adopt the first and more familiar approach with $m_{4}=0$.

A crucial aspect of this theory is how the anomaly prevents rotations between $m_{1} \bar{\psi} \psi$ and $\operatorname{im}_{3} \bar{\psi} \gamma_{5} \psi$. Such would follow from a hypothetical variable change

$$
\psi \rightarrow e^{i \theta \gamma_{5}} \psi
$$

This however is not a valid symmetry $[13,14,15,16]$ because it changes the fermion measure

$$
d \psi \rightarrow e^{i \theta \operatorname{Tr} \gamma_{5}} d \psi
$$

The issue, as nicely elucidated by Fujikawa [17], is that in any regulated theory $\gamma_{5}$ cannot remain traceless. For example, consider a cutoff $\Lambda$ and regulate the theory suppressing large eigenvalues of the Dirac operator $D$. The index theorem gives the result

$$
\operatorname{Tr} \gamma_{5} e^{D^{2} / \Lambda^{2}}=v
$$

where $v$ is the winding number of the gauge field configuration under consideration. Thus the above rotation will introduce a factor of $\exp (i \theta v)$ into the path integral and thereby change the value of the QCD Theta parameter. Actually, the above rotation allows one to move any Theta parameter from the gauge action into the mass terms. For the following, assume that this has been done. After this, all three mass parameters are both relevant and independent. 


\section{The strong CP problem}

Experimentally the strong interactions preserve CP symmetry to high accuracy. This would not be the case if $m_{3}$ were substantial. Indeed, only the two parameters $m_{1}$ and $m_{2}$ seem to be needed. The strong $\mathrm{CP}$ problem asks why is $m_{3}$ so small?

This issue arises because of the possible unification of interactions. The weak interactions are known to violate $\mathrm{CP}$; so, when the interactions separate as one goes down in energy, why is it that some residue of the CP violation doesn't remain in a non-vanishing $m_{3}$.

One trivial "solution" is that there is no unification. One could consider the strong interactions on their own and impose CP symmetry from the outset. In this picture the weak interactions only come in as a small perturbation and do not directly affect the Theta angle.

Another approach couples a new dynamical field directly to $i \bar{\psi} \gamma_{5} \psi$. In this case $m_{3}$ becomes a dynamical quantity and can relax naturally to zero. This requires a new particle corresponding to this field, although its coupling is not determined and could be small. This is the "axion" approach.

It is sometimes proposed that the strong CP problem could be solved by having the up quark mass vanish. However the above formalism should clarify why this is not a sensible approach. In terms of the three mass variables, one could define the up quark mass as

$$
m_{u} \equiv m_{1}+m_{2}+i m_{3}
$$

The problem is that $m_{1}, m_{2}$, and $m_{3}$ are independent parameters with different symmetry properties. The parameter $m_{1}$ represents an isosinglet mass contribution while $m_{2}$ multiplies an isovector quantity. It is only the parameter $m_{3}$ which is CP violating. And the discussion in the introduction showed that $m_{1}+m_{2}=0$ is a scale and scheme dependent statement. So while it may be true that setting $m_{u}$ from Eq. (4.1) to zero would imply $m_{3}=0$, this could be regarded as "not even wrong."

The basic issue with forcing the up quark mass to zero is that it involves going to polar coordinates with an unnatural origin. In a formal sense one can connect the three mass parameters above with the more conventional set $\left\{m_{u}, m_{d}, \Theta\right\}$ via the relations

$$
\begin{gathered}
m_{u}=m_{1}+m_{2}+i m_{3}, \\
m_{d}=m_{1}-m_{2}+i m_{3}, \\
e^{i \Theta}=\frac{m_{1}^{2}-m_{2}^{2}-m_{3}^{2}+2 i m_{1} m_{3}}{\sqrt{m_{1}^{4}+m_{2}^{4}+m_{3}^{4}+2 m_{1}^{2} m_{3}^{2}+2 m_{2}^{2} m_{3}^{2}-2 m_{1}^{2} m_{2}^{2}}} .
\end{gathered}
$$

The mixing discussed in the introduction shows that this choice of parameters, including $\Theta$, is in general scale and scheme dependent.

\section{The phase diagram}

Taking the mass parameters away from their physical values uncovers a rather rich phase diagram. This follows from a simple linear sigma model analysis. For this, consider the composite scalar fields

$$
\sigma \propto \bar{\psi} \psi, \quad \vec{\pi} \propto i \bar{\psi} \gamma_{5} \vec{\tau} \psi, \quad \eta \propto i \bar{\psi} \gamma_{5} \psi, \quad \vec{a}_{0} \propto \bar{\psi} \vec{\tau} \psi
$$




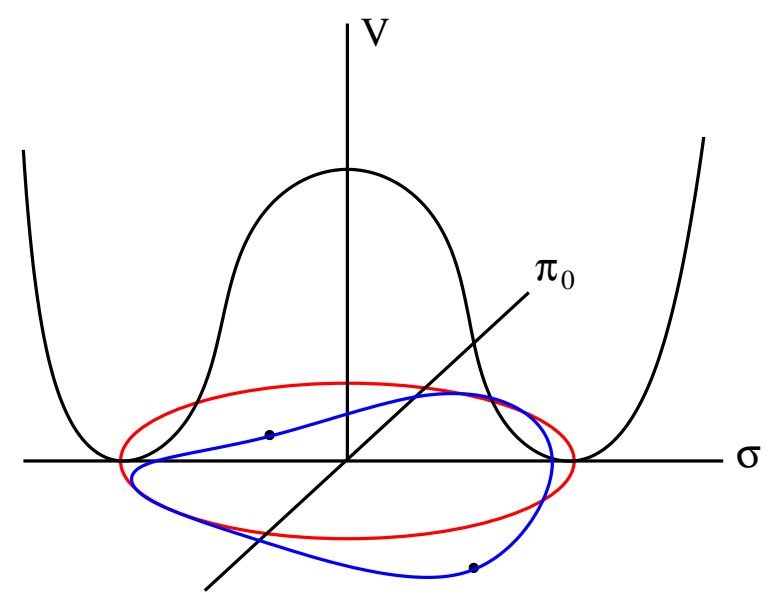

Figure 3: The parameters $m_{2}$ and $m_{3}$ warp the Mexican hat downward in a direction determined by their relative size.

These provide a model for the two flavor chiral symmetry via an effective potential

$$
\begin{gathered}
V=\lambda\left(\sigma^{2}+\vec{\pi}^{2}-v^{2}\right)^{2}-m_{1} \sigma-m_{2} a_{03}-m_{3} \eta \\
+\alpha\left(\eta^{2}+\vec{a}_{0}^{2}\right)-\beta\left(\eta \sigma+\vec{a}_{0} \cdot \vec{\pi}\right)^{2} .
\end{gathered}
$$

The first term, proportional to $\lambda$, is the conventional "wine bottle" or "Mexican hat" frequently used to describe spontaneous symmetry breaking. The parameters $\alpha$ and $\beta$ can be thought of as "low energy constants" that couple $(\sigma, \vec{\pi})$ with $\left(\eta, \vec{a}_{0}\right)$. These combinations rotate similarly under flavored chiral rotations; so, these constants preserve the chiral symmetry of the massless theory. Here the $\alpha$ term serves to give a mass to the $\eta$ and $\vec{a}_{0}$. The square appearing in the $\beta$ term is inserted so the basic potential still preserves parity. The sign of this term is selected so that $m_{\eta}<m_{\vec{a}_{0}}$.

The three mass terms break the chiral symmetry in slightly different ways. The $m_{1}$ term serves to tilt this potential and generally selects a unique minimum. The effects of the $m_{2}$ and $m_{3}$ terms are more subtle since they do not directly couple to the $\pi$ or $\sigma$ fields. With $m_{2}\left(m_{3}\right)$ present the field $a_{03}(\eta)$ will be driven to have an expectation value. This will feed back through the $\beta$ term to give a quadratic warping of the Mexican hat. This warping will be downward in the $\pi_{0}(\sigma)$ direction. With both terms present, this warping will be in some intermediate direction, as shown in Fig. 3. When $m_{1}$ is absent, this warping leaves two possible minima into which the vacuum can settle. Turning on a small $m_{1}$, the resulting tilt will select one or the other as the true vacuum. This results in a generic first order transition occuring when $m_{1}$ changes sign.

A special case occurs when $m_{3}=0$ and $m_{2} \neq 0$. Then the warping is downward in the $\pi_{0}$ direction and $m_{1}$ does not distinguish between the two minima, as sketched in Fig. 4 . In this situation there will be some intermediate value of $m_{1}$ where a single minimum at large tilt splits into two minima with an expectation value for the neutral pion field. This is sketched in Fig. 5. At this critical point one expects an Ising-like behavior. Here the square of the neutral pion mass passes through zero and gives rise to a pion condensate. As the pion is CP odd, this represents a spontaneous breaking of CP symmetry.

Note that this Ising-like transition at $m_{3}=0,\left|m_{1}\right|<\left|m_{2}\right|$ occurs with both $m_{u}$ and $m_{d}$ nonvanishing, although they are of opposite sign. This represents a situation where there is a diverging 


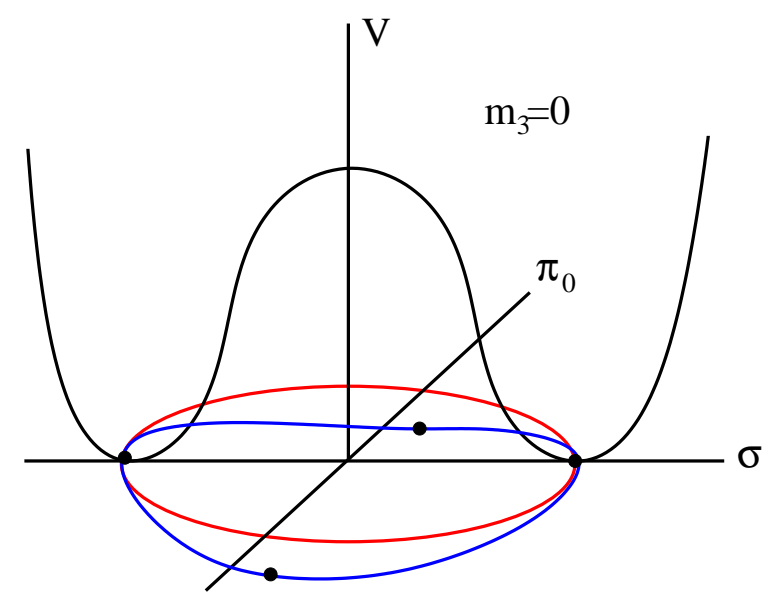

Figure 4: At $m_{3}=0$ the warping is orthogonal to the sigma direction and a small $m_{1}$ term does not select a unique minimum.
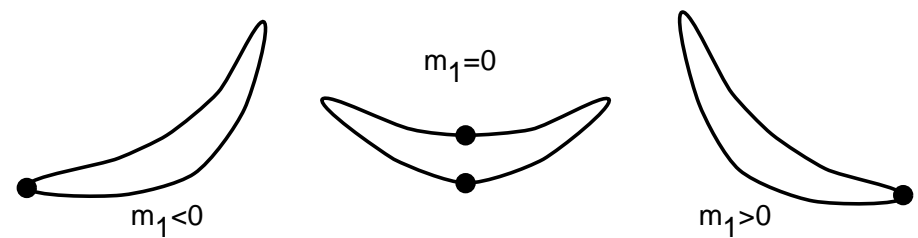

Figure 5: As $m_{1}$ varies at $m_{3}=0$ there should be a point where a single minimum splits into two.

correlation length and corresponding long distance physics occuring without the presence of any small eigenvalues for the Dirac operator.

Conversely, the overall picture indicates no special behavior at $m_{u}=0$ when $m_{d} \neq 0$. In this case there is no important long distance physics despite the possibility of small Dirac eigenvalues. These facts are the seed of many controversies, including the connection between the strong $\mathrm{CP}$ problem and $m_{u}=0$ [7], the issue of whether topological susceptibility is a physical observable [18], and the failure of the rooting process for staggered fermions [19].

The final phase diagram as a function of the three mass parameters appears in Fig. 6. There are two intersecting first order surfaces, one at $\left(m_{1}=0, m_{3} \neq 0\right)$ and the second contained in the region $\left(m_{1}<m_{2}, m_{3}=0\right)$. The second surface ends along a critical line. In conventional language, these transitions all occur when the strong CP angle takes the value $\pi$, but it is important to note that there is a finite region with $\Theta=\pi$ without any phase structure, i.e. when $m_{2}$ is only slightly larger than $m_{1}$. Here the quark masses differ in sign, but one is much smaller than the other in magnitude. 


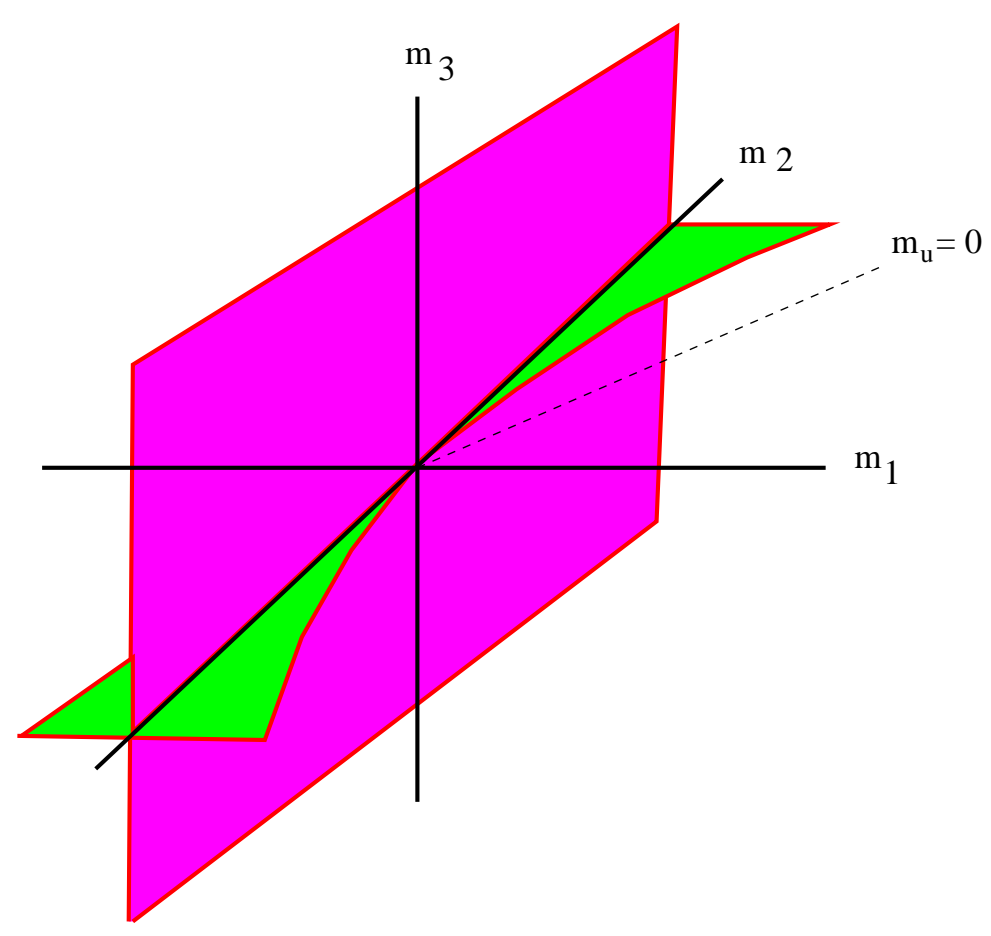

Figure 6: The full phase diagram as a function of the three mass parameters.

\section{Summary}

Non-perturbative effects can result in a mixing between the masses for different quark species. Because this effect is absent in perturbation theory, it is inappropriate to match lattice and perturbative calculations of quark masses, particularly when they are non-degenerate.

The two flavor theory depends on three possible mass parameters. One of these is explicitly CP violating; its apparent absence is the strong $\mathrm{CP}$ problem. As these three parameters are varied from their physical values, a rather rich phase diagram is encountered, displaying both first and second order transitions. In this diagram there is no structure at $m_{u}=0$ when $m_{d} \neq 0$. This is closely connected with the result that $m_{u}=0$ is not an appropriate solution to the strong CP problem. 


\section{References}

[1] Michael Creutz. Anomalies and discrete chiral symmetries. PoS, QCD-TNT09:008, 2009.

[2] Michael Creutz. Quark mass dependence of two-flavor QCD. Phys. Rev., D83:016005, 2011.

[3] Michael Creutz. Confinement, chiral symmetry, and the lattice. Acta Physica Slovaca, 61:1-127, 2011.

[4] Howard Georgi and Ian N. McArthur. Instantons and the $m_{u}$ Quark Mass. unpublished (HUTP-81/A011), 1981.

[5] Tom Banks, Yosef Nir, and Nathan Seiberg. Missing (up) mass, accidental anomalous symmetries, and the strong CP problem. unpublished (hep-ph/9403203), 1994.

[6] Michael Creutz. CP symmetry and the strong interactions. unpublished (hep-th/0303254), 2003.

[7] Michael Creutz. Ambiguities in the up quark mass. Phys. Rev. Lett., 92:162003, 2004.

[8] A. Bazavov, D. Toussaint, C. Bernard, J. Laiho, C. DeTar, et al. Nonperturbative QCD simulations with 2+1 flavors of improved staggered quarks. Rev.Mod.Phys., 82:1349-1417, 2010.

[9] T. Blum, R. Zhou, T. Doi, M. Hayakawa, T. Izubuchi, et al. Electromagnetic mass splittings of the low lying hadrons and quark masses from 2+1 flavor lattice QCD+QED. Phys.Rev., D82:094508, 2010.

[10] S. Durr, Z. Fodor, C. Hoelbling, S.D. Katz, S. Krieg, et al. Lattice QCD at the physical point: light quark masses. Phys.Lett., B701:265-268, 2011.

[11] R. Frezzotti and G.C. Rossi. Chirally improving Wilson fermions. 1. O(a) improvement. JHEP, 0408:007, 2004.

[12] Gernot Munster, Christian Schmidt, and Enno E. Scholz. Chiral perturbation theory for twisted mass QCD. Nucl.Phys.Proc.Suppl., 140:320-322, 2005.

[13] Stephen L. Adler. Axial vector vertex in spinor electrodynamics. Phys.Rev., 177:2426-2438, 1969.

[14] Stephen L. Adler and William A. Bardeen. Absence of higher order corrections in the anomalous axial vector divergence equation. Phys.Rev., 182:1517-1536, 1969.

[15] J.S. Bell and R. Jackiw. A PCAC puzzle: pi0 to gamma gamma in the sigma model. Nuovo Cim., A60:47-61, 1969.

[16] Roman Jackiw. What good are quantum field theory infinities? 1999. hep-th/9911071.

[17] Kazuo Fujikawa. Path integral measure for gauge invariant fermion theories. Phys.Rev.Lett., 42:1195, 1979.

[18] Michael Creutz. Anomalies, gauge field topology, and the lattice. Annals Phys., 326:911-925, 2011.

[19] Michael Creutz. Chiral anomalies and rooted staggered fermions. Phys. Lett., B649:230-234, 2007. 